Proceedings of the 43rd "Jaszowiec", International School and Conference on the Physics of Semiconductors, Wisła 2014

\title{
Application of the Dot-Ring Nanostructure to Control Electrical Transport in the Coulomb Blockade Regime
}

\author{
I. Janus-Zygmunt, B. Kędzierska, A. Gorczyca-Goraj, M. Kurpas, M.M. Maśka \\ AND E. ZIPPER
}

Department of Theoretical Physics Institute of Physics, University of Silesia, Uniwersytecka 4, 40-007 Katowice, Poland

\begin{abstract}
Transport properties of a two-dimensional nanostructure composed of a quantum dot surrounded by a quantum ring (dot-ring nanostructure), are discussed. This complex system is a highly controllable object. Conduction through dot-ring nanostructure depends crucially on the coupling strength of its states to the electrodes, which is related to the spatial distribution of the electron's wave functions in dot-ring nanostructure. This distribution can be strongly modified, e.g., by the electrical gating so that the ground and excited states move between the inner dot and the outer ring. In this paper we show that this property can be used to control single-electron DC current through dot-ring nanostructure in the Coulomb blockade regime so that it can be used as a single electron transistor.
\end{abstract}

DOI: $10.12693 /$ APhysPolA.126.1171

PACS: 73.23.Hk, 73.21.La, 73.22.-f

\section{Introduction}

Quantum nanostructures (QNs) [1, 2] exhibit new physics which has no analogue in real atoms. In particular, the electronic properties of QNs can be finely tuned adjusting structural parameters such as size and shape. The latter parameter is particularly important as its small variations can cause dramatic changes of the electronic properties [3]. Thus especially interesting in this context are complex nanostructures.

The purpose of this study is to demonstrate a highly controllable transport properties of a two-dimensional nanostructure in the form of a quantum dot (QD) surrounded by a quantum ring (QR), named afterwords a dot-ring nanostructure (DRN). Such structures have been recently fabricated [4]. By changing the confinement potential, e.g., by the electrical gating one can change the shape and distribution of the electron wave functions which determine many physical, measurable quantities $[5,6]$.

Conduction through DRN is strongly related to the coupling strength of its states to the electrodes which, in turn, depends on the localization of the electron's wave function: states localized in QD (QR) are weakly (strongly) coupled to the electrodes. We show that this property can be used to control single-electron DC current through DRN in the Coulomb blockade regime. In particular, we show that one can adjust the confinement potential of DRN so that it can be used as a single electron transistor.

\section{Model and assumptions}

We consider a 2D, circularly symmetric dot-ring nanostructure defined by a confinement potential $V(r)$ occupied by a single electron. The DRN is composed of a QD surrounded by a QR and separated from the ring by a potential barrier $V_{0}(r)$ which allows the electron tunneling between the QD and QR. A cross section of a DRN with explanation of symbols used throughout the text is presented in Fig. 1.

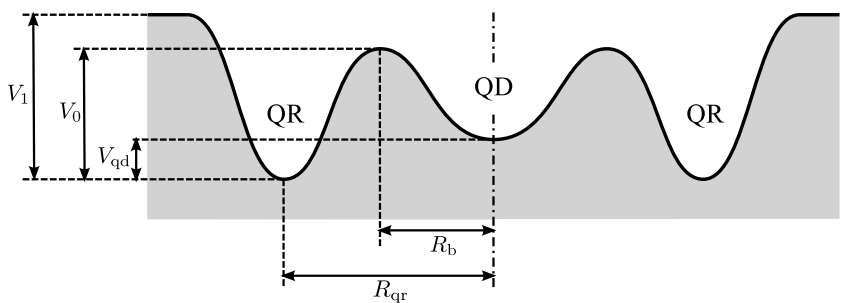

Fig. 1. Cross section of the confining potential $V(r)$ of a DRN. The potential is parameterized under the assumption that all values are measured with respect to the bottom of the QR part of the DRN. $V_{\mathrm{qd}}, V_{0}$ and $V_{1}$ denote the bottom the QD part, the height of the potential barrier between the $\mathrm{QD}$ and $\mathrm{QR}$, and the value of the potential outside the DRN, respectively. $R_{\mathrm{b}}$ and $R_{\mathrm{qr}}$ denote the radii of the barrier and the $Q R$, respectively.

In our model calculations we take the radius of DRN $r_{0}=80 \mathrm{~nm}, V_{1}=90 \mathrm{meV}$ and set the zero potential energy at the level of $V_{\mathrm{qr}}$, i.e., the potential well offset is equal to $V_{\mathrm{qd}}$. Such a confinement potential, which conserves the circular symmetry [7], can be obtained in many ways, e.g., using atomic force microscope to locally oxidize the surface of a sample [8], by self-assembly techniques (in particular by pulsed droplet epitaxy) [4, 9], by the split gates [10], or by lithography.

We solve numerically the Schrödinger equation assuming the Gaussian form of the confining potential [5]. The energy spectrum of the system consists of a set of discrete states $E_{n l}$ due to radial motion with radial 
quantum numbers $n=0,1,2, \ldots$, and rotational motion with angular momentum quantum numbers $l=$ $0, \pm 1, \pm 2 \ldots$ The single particle orbital wave function is of the form

$$
\Psi_{n l \sigma}=R_{n l}(r) \exp (\mathrm{i} l \phi),
$$

with the radial part $R_{n l}(r)$. We neglect here the spin degrees of freedom; the spin dependent transport will be discussed elsewhere.

In the considered case the electron states and their wave functions are known exactly but they strongly depend on the confinement $V(r)$ which can be modified, e.g., by electrical gating. In Fig. 2 we show the distribution of the wave functions of the ground and exited states for different values of $V_{\text {qd }}$.
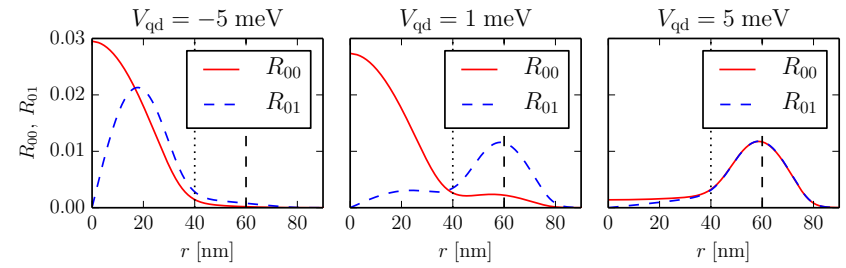

Fig. 2. Illustration of the wave function engineering: for $V_{\mathrm{qd}}=-5 \mathrm{meV}$ both the ground and the first excited state wave functions are located in QD, for $V_{\mathrm{qd}}=1 \mathrm{meV}$ the ground state wave function remains in $\mathrm{QD}$, but the wave function of the first excited states is moved to $\mathrm{QR}$, and finally for $V_{\mathrm{qd}}=5 \mathrm{meV}$ both the wave functions are in QR. In all cases $V_{0}=15 \mathrm{meV}$ has been assumed. The dotted and dashed vertical lines indicate $R_{\mathrm{b}}$ and $R_{\mathrm{qr}}$, respectively.

We see that the localization of the individual wave functions of the ground and excited states changes between the QD and the QR parts of DRN when we change $V_{\mathrm{qd}}$ from $V_{\mathrm{qd}}<0$ to $V_{\mathrm{qd}}>0$. Such manipulations can be done experimentally by the application of a disk-shaped potential gate below the inner QD.

In this work we calculate the single-electron DC current through DRN in the Coulomb blockade regime near the $N=0 \leftrightarrow 1$ transition. It means that one can transit only a single electron at a time through the structure, while the higher order tunneling events are neglected [11]. The second electron cannot enter because it would have cost the Coulomb energy $E_{\mathrm{C}},\left|\mu_{\mathrm{S}}-\mu_{\mathrm{D}}\right| \leq E_{\mathrm{C}}$. Since in our studies both the energy spacing of the DRN states and the source drain bias are much larger than the thermal energy, we take $T=0$.

\section{DRN as a single electron transistor}

The system we study is the DRN weakly coupled to the source and drain electrodes. $\Gamma_{\mathrm{S}}, \Gamma_{\mathrm{D}}$ denote the subsequent tunnel rates. In this chapter we focus on the case of equal tunnel barriers, i.e., $\Gamma_{\mathrm{S}}=\Gamma_{\mathrm{D}}=\Gamma$. We also assume that only the ground state is in the bias window, $\mu_{\mathrm{D}} \leq E_{\mathrm{G}} \leq \mu_{\mathrm{S}}$. The current in the pure QD case exhibits then the current peak [2]. However in the case of
DRN we can manipulate the confinement so that we obtain the transistor behavior: if the electron is located in the inner QD the conduction through DRN will be small due to negligible couplings $\Gamma$, whereas when the electron is located in the outer QR the conduction will be high due to large $\Gamma$ 's. We have investigated different sets of parameters to get proper transistor behavior. The DC current $I$ is calculated from the formula

$$
I=-e \frac{\Gamma_{\mathrm{S}} \Gamma_{\mathrm{D}}}{\Gamma_{\mathrm{S}}+\Gamma_{\mathrm{D}}}=-e \frac{\Gamma}{2} \text { for } \Gamma_{\mathrm{S}}=\Gamma_{\mathrm{D}},
$$

where $-e$ is the electron charge.

Many investigations of transport properties make use of the high tunability of the tunnel barriers of the electrode by voltage pulses [11]. In our analysis we utilize instead the high tunability of the electron states in DRN while keeping the barrier parameters constant. The tunnel rate $\Gamma$ depends on the overlap between the DRN wave function and the wave function of the electrode which can be strongly modified by changing the potential $V_{\mathrm{qd}}$ - the bottom of the inner QD and the height of the barrier $V_{0}$. The $\Gamma$ 's have been calculated by us microscopically using the method proposed in [12].
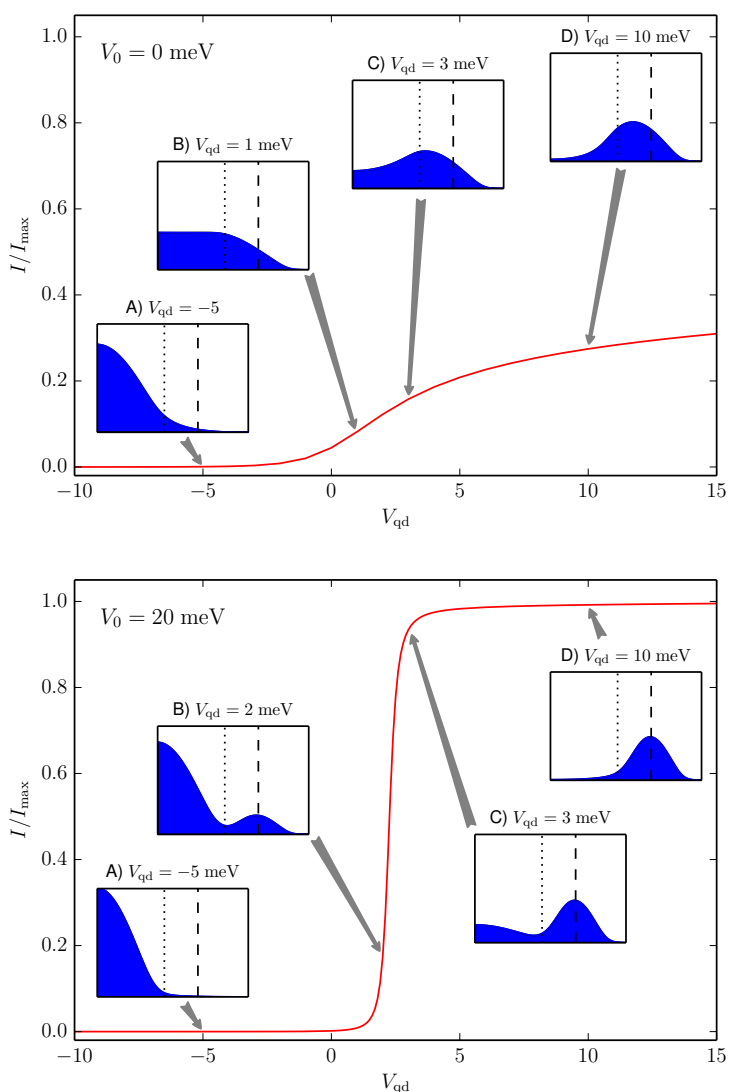

Fig. 3. Current through a DRN as a function of the position of bottom of the QD potential $V_{\mathrm{qd}}$ for the barrier height $V_{0}=0$ (upper part) and $V_{0}=20$ (lower part). The insets show the radial part of the corresponding wave functions for different $V_{\mathrm{qd}}$. The dotted and dashed vertical lines indicate $R_{\mathrm{b}}$ and $R_{\mathrm{qr}}$, respectively. 
In Fig. 3 we present the results of the calculations of the current as a function of $V_{\mathrm{qd}}$ for $V_{0}=0$ and $V_{0}=20 \mathrm{meV}$. The distribution of the ground state wave functions for different values of the parameters is shown in the insets. We see that in the absence of the barrier $V_{0}=0$ the increase of the current is rather small. In this case increasing $V_{\text {qd }}$ gradually pushes the ground state wave function towards the outer parts of the DRN thus increasing the coupling between the ground state and the electrodes. However, the steepness of the $I-V_{\mathrm{qd}}$ characteristics is significantly larger for $V_{0}=20 \mathrm{meV}$. In this case the wave function of the ground state cannot smoothly evolve from the QD to the QR parts of the DRN. Instead, for some critical value of $V_{\mathrm{qd}}$ it is suddenly transferred through the barrier, which results in a sudden increase of the current. The switching is even faster for larger values of $V_{0}$.

\section{Conclusions}

One of the important properties of DRN is the high controllability of the shapes of the electron wave functions. We have shown that this very feature offers the possibility to build a single electron transistor based on different physical mechanism than in ordinary QDs. Namely, by placing the electron ground state in the bias window one can regulate the current by changing the location of the wave function by electrical gating. Such complex systems with a single or a few electrons trapped are potentially useful for nanoelectronics as they require very little energy to supply.

\section{Acknowledgments}

This work was supported by the Polish National Science Centre (NCN) under grant DEC2013/11/B/ST3/00824.

\section{References}

[1] R. Hanson, D.D. Awschalom, Nature 453, 1043 (2008).

[2] R. Hanson, L.P. Kouwenhoven, J.R. Petta, S. Tarucha, L.M.K. Vandersypen, Rev. Mod. Phys. 79, 1217 (2007).

[3] S. Amasha, K. MacLean, P. Iuliana, D.M. Zumbühl, M.A. Kastner, M.P. Hanson, A.C. Gossard, Phys. Rev. Lett. 100, 046803 (2008).

[4] C. Somaschini, S. Bietti, N. Koguchi, S. Sanguinetti, Nanotechnology 22, 185602 (2011).

[5] E. Zipper, M. Kurpas, M.M. Maśka, New J. Phys. 14, 093029 (2012).

[6] M. Kurpas, E. Zipper, M.M. Maśka, in: Physics of Quantum Rings, Ed. V.M. Fomin, Springer, 2014, p. 455 .

[7] B. Szafran, F.M. Peeters, S. Bednarek, Phys. Rev. $B$ 70, 125310 (2004).

[8] A. Fuhrer, S. Lscher, T. Ihn, T. Henzel, K. Ensslin, W. Wegscheider, M. Bichler, Nature 413, 822 (2001).

[9] T. Mano, T. Kuroda, K. Mitsuishi, M. Yamagiwa, X.-J. Guo, K. Furuya, K. Sakoda, N. Koguchi, J. Cryst. Growth 301, 740 (2007); T. Kuroda, T. Mano, T. Ochiai, S. Sanguinetti, K. Sakoda, G. Kido, N. Koguchi, Phys. Rev. B 72, 20530 (2005).

[10] N.B. Zhitenev, M. Brodsky, R.C. Ashoori, L.N. Pfeiffer, K.W. West, Science 285, 715 (1999).

[11] R. Hanson, I.T. Vink, D.P. DiVincenzo, L.M.K. Vandersypen, J.M. Elzerman, L.H. Willems van Beveren, L.P. Kouwenhoven, in: Proc. 39th Rencontres de Moriond, 2004.

[12] J. Bardeen, Phys. Rev. Lett. 6, 57 (1961). 